\title{
Mimicking Cellular Signaling Pathways within Synthetic Multicompartment Vesicles with Triggered Enzyme Activity and Induced Ion Channel Recruitment
}

\author{
Sagana Thamboo, Adrian Najer, Andrea Belluati, Claudio von Planta, Dalin Wu, \\ loana Craciun, Wolfgang Meier,* and Cornelia G. Palivan*
}

Subcellular compartmentalization of cells, a defining characteristic of eukaryotes, is fundamental for the fine tuning of internal processes and the responding to external stimuli. Reproducing and controlling such compartmentalized hierarchical organization, responsiveness, and communication is important toward understanding biological systems and applying them to smart materials. Herein, a cellular signal transduction strategy (triggered release from subcompartments) is leveraged to develop responsive, purely artificial, polymeric multicompartment assemblies. Incorporation of responsive nanoparticles-loaded with enzymatic substrate or ion channels-as subcompartments inside micrometersized polymeric vesicles (polymersomes) allowed to conduct bioinspired signaling cascades. Response of these subcompartments to an externally added stimulus is achieved and studied by using confocal laser scanning microscopy (CLSM) coupled with in situ fluorescence correlation spectroscopy (FCS). Signal triggered activity of an enzymatic reaction is demonstrated in multicompartments through recombination of compartmentalized substrate and enzyme. In parallel, a two-step signaling cascade is achieved by triggering the recruitment of ion channels from inner subcompartments to the vesicles' membrane, inducing ion permeability, mimicking endosome-mediated insertion of internally stored channels. This design shows remarkable versatility, robustness, and controllability, demonstrating that multicompartment polymer-based assemblies offer an ideal scaffold for the development of complex cell-inspired responsive systems for applications in biosensing, catalysis, and medicine.

\section{Introduction}

The spatiotemporal separation of biochemical reactions plays a fundamental role in nature: their compartmentalization allows the steering of reaction kinetics via controlled diffusion through specialized microenvironments, while also protecting their content from degrading agents or generally incompatible compounds. ${ }^{[1]}$ In nature, compartments can either be horizontal, with no clear directionality in their communication and regulation (cell colonies or tissues), or hierarchical, where one compartment controls other lower-tier, often encapsulated, subcompartments, achieving a higher organizational level (organelles inside cell). ${ }^{[2,3]}$ In addition, natural compartments are often stimuli-responsive, an essential trait of living cells, enabling them to adapt their developmental or metabolic state based on external conditions or to communicate through signaling cascades. The control of cellular signaling pathways includes changes in protein activity, protein concentration, protein-protein interactions, and protein localization. ${ }^{[4]}$ With respect to the latter, triggered recruitment of cellular receptors, transporters, and ion channels from internal compartments (early

S. Thamboo, Dr. A. Najer, ${ }^{[+]}$A. Belluati, Dr. D. Wu,

Dr. I. Craciun, Prof. W. Meier, Prof. C. G. Palivan

Department of Chemistry

University of Basel

Mattenstrasse 24a, BPR 1096, 4058 Basel, Switzerland

E-mail:wolfgang.meier@unibas.ch; cornelia.palivan@unibas.ch

C. von Planta

Department of Chemistry

University of Basel

Klingelbergstrasse 80, 4056 Basel, Switzerland

The ORCID identification number(s) for the author(s) of this article can be found under https://doi.org/10.1002/adfm.201904267.

${ }^{[+]}$Present address: Department of Materials, Imperial College London, South Kensington Campus, Prince Consort Road, London SW7 2AZ, UK.

DOI: 10.1002/adfm.201904267 endosomes or recycling endosomes) to the cell membrane and vice versa is important to control and fine-tune cellular signaling, as has been described for neurons ${ }^{[5,6]}$ and cardiac muscle cells. ${ }^{[7,8]}$

Due to their importance in nature, both compartmentalization, at the micro and nanoscale, and stimuli-responsiveness are a source of inspiration for development of advanced functional materials. ${ }^{[3]}$ Giant lipid vesicles (giant liposomes, from 1 to $200 \mu \mathrm{m}$ ) are still widely used thanks to their biocompatibility as cellular models; however, the number of membrane forming lipids is limited, and they have a lower stability and limited chemical versatility compared to synthetic membranes. ${ }^{[9,10]}$ Block copolymers provide conditions to overcome these limitations because, depending on the polymer of choice, they offer a vast chemical versatility, mechanical stability, and allow tuning 
of the membrane properties, in addition to biocompatibility or biodegradability. ${ }^{[9,11]}$ In particular, stimuli-responsive single polymer-based nanocompartments were proposed as drug delivery systems, ${ }^{[12]}$ nanoreactors, ${ }^{[13]}$ artificial organelles ${ }^{[14]} /$ cells, ${ }^{[15]}$ depending on their payload and the properties of their membrane. Stimuli-responsiveness was achieved either by designing copolymers with a special chemical nature, inducing a change in the membrane permeability ${ }^{[16]}$ or even its disintegration $^{[17]}$ upon a change in their environment. Alternatively, functional insertion of ion channels (biopores) and membrane proteins allowed induction of membrane permeability owing to their intrinsic functionality. ${ }^{[18]}$

On the other hand, multicompartments based on hierarchical compartment organization have been introduced with a long-term goal of designing simple artificial cells as tools to better understand natural mechanisms and provide innovative solutions for biomedical and chemical challenges. ${ }^{[19,20]}$ The spatiotemporal interdependence of reactions is also the basis of molecular logics, where compounds are used to process physicochemical inputs and produce outputs based on logical operators, aiming to develop nonelectronic computation. ${ }^{[21,22]} \mathrm{A}$ microscale reactor capable of predictable self-regulation would thus be an important step in the development of materials with a smart behavior.

Multicompartments have been developed in a variety of setups by using different combinations of lipid membranes, ${ }^{[23]}$ (in)organic capsules, ${ }^{[19,24,25]}$ amphiphilic polymer membranes, ${ }^{[23,26]}$ and multilayer polyelectrolyte membranes. ${ }^{[27]}$ For example, simple responsive cellular mimics were proposed using capsosome-based compartments loaded with stimuliresponsive adamantyl-modified polymersomes, ${ }^{[28]}$ capsules containing $\mathrm{pH}$-dependent liposomes, ${ }^{[29]}$ lipid giant vesicles loaded with UV-responsive liposomes, ${ }^{[30]}$ and microscale polymersomes encapsulated with temperature-dependent liposomes to trigger specific chemical reactions. ${ }^{[23]}$
In addition, microfluidic techniques promise high encapsulation efficiency inside microcompartments, precise operation capability and size monodispersity, ${ }^{[31,32]}$ but up to now, their production rates are low and with reduced diversity. ${ }^{[33]}$ Despite these promising examples, the potential of microscale multicompartments as novel functional materials is still restricted by a limited implementation of responsiveness to external stimuli, not taking advantage of the time dimension to modify the behavior and architecture of such multicompartments in a controlled manner.

Here we present how polymeric stimuli-responsive multicompartment vesicles can be designed to self-regulate their activity in a bioinspired manner, which mimics cellular signaling pathways. We also highlight the modularity of our system, giving us a truly flexible multipurpose tool that can be readily adapted for multiple functions. We start with a multicompartment architecture based on synthetic vesicles with sizes in the micrometer range (giant unilamellar vesicles, GUVs) loaded with synthetic subcompartments and induce their functionality through a stimulus present in their environment (Scheme 1A). To achieve the environmental responsiveness, the loaded subcompartments are reduction-sensitive thus possessing a triggerable activity resulting in a morphological change of the microcompartments. The multicompartments are formed by one-pot self-assembly of a mixture of amphiphilic block copolymers poly(2-methyl-2-oxazoline) $)_{5}$-blockpoly(dimethylsiloxane) ${ }_{58}$-block-poly (2-methyl-2-oxazoline) ${ }_{5}$ $\left(\mathrm{PMOXA}_{5}-b\right.$ - $\mathrm{PDMS}_{58}-b$ - $\mathrm{PMOXA}_{5}$ ) and $\mathrm{PDMS}_{65}-b$-heparin in the presence of specific molecules (enzymes, reporter compounds) and prior formed stimuli-responsive subcompartments, which contain the enzyme substrates or ion channels. Spatial segregation inside synthetic GUVs is realized by simultaneous coencapsulation of desired molecules, and respective partners entrapped inside subcompartments. $\mathrm{PDMS}_{65}-b$-heparin served to equip the multicompartment surface with a biological

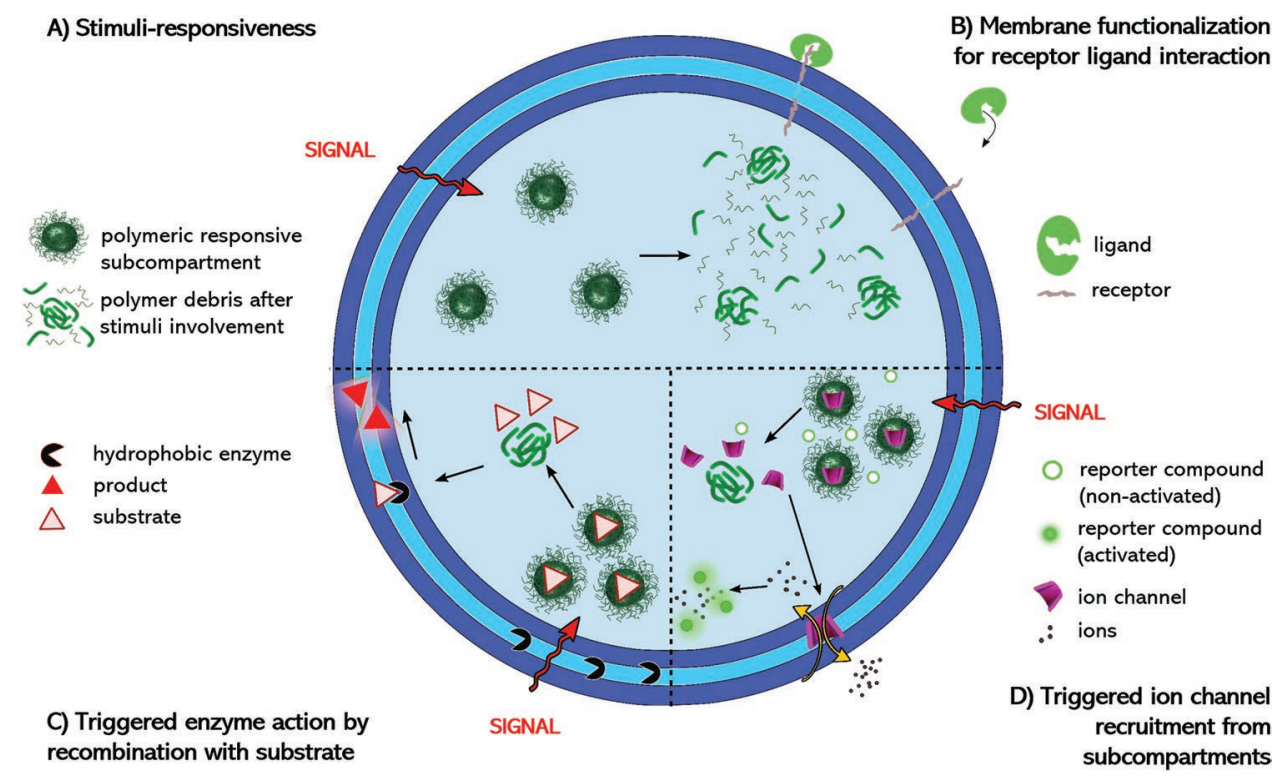

Scheme 1. Schematic representation of our modular multicompartment system composed of reduction sensitive subcompartments for triggered enzymatic activity and ion channel recruitment, encapsulated within polymeric giant unilamellar vesicles. The reducing agent DTT was used as the signal (red arrow) that passively traverses the polymeric GUV membrane without addition of biopores or channels. 
receptor-like moiety that will further support possible bio-interactions (Scheme 1B). ${ }^{[34-36]}$

A sequential setup provides the multicompartments' activity: production of desired molecules by in situ triggered enzymatic reactions using the mainly hydrophobic enzyme lipase in the GUV membrane (Scheme 1C), or selective flow of ions in/ out via ion channel recruitment in the GUVs' membrane as a molecular AND gate (Scheme 1D) for biosensing purposes. Due to hydrophobic forces, the ion channel gramicidin will auto-integrate into the polymer membrane after release from subcompartments, as previously demonstrated when directly added to pre-formed polymersomes. ${ }^{[37]}$

An external signaling molecule (dithiothreitol, DTT) added to the environment of the multicompartments passively diffuses across the GUVs' membrane, inducing the disassembly of the reduction-sensitive nanoparticles and the release of the entrapped cargo. Depending on their chemical nature (enzyme substrates or biopores), these molecules initiate either an enzymatic reaction or a selective ion flow through the GUVs membrane. On the contrary, when the subcompartments are non-responsive or the external stimulus is not present, the multicompartment stays intact. The combination of a primary signal (presence of stimulus in the environment of multicompartments) with a secondary one (induced ion flow to/from the environment) represents a straightforward model that can be used to study ion channel recruitment from internal subcompartments to the membrane upon cellular signaling, a process naturally occurring in neuronal cells. ${ }^{[5,6]}$ Triggered activity and change in the architecture of synthetic multicompartment vesicles, in auto-controlled sequences, are expected to open new directions for applications of such functional systems in domains such as medicine, catalysis, and biosensing.

\section{Results and Discussion}

\subsection{Multicompartment Self-Assembly: Loading Synthetic Giant Vesicles with Subcompartments}

The production of multicompartmentalized synthetic giant unilamellar vesicles that expose receptor-like molecules on the polymer membrane and contain subcompartments, was achieved by formation of GUVs loaded with prior self-assembled nanoscale assemblies (polymersomes, nanoparticles, and micelles) (Figure 1A). GUVs were self-assembled from a mix-

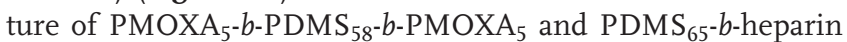
copolymers $(8 \mathrm{wt} \%),{ }^{[34]}$ the latter negatively charged copolymer serving to expose heparin on the GUV membrane as a mimic for heparan sulfate, known to be exposed on the plasma membrane of most cell types. ${ }^{[38]}$

First, we investigated the self-assembly of empty GUVs formed by gentle film rehydration method in order to optimize formation of unilamellar vesicles. After drying the copolymer film under vacuum overnight to eliminate traces of organic solvents, a rehydration solution was added to induce the self-assembly process, and confocal laser scanning microscopy (CLSM) was used to characterize the resulting system (Figure 1B). When used as a rehydration solution, water and PBS buffer generated a mixture of various assemblies, ranging from multilamellar vesicles up to polymer junk and filled assemblies (Figure S1B,C, Supporting Information). According to CLSM micrographs, the use of increasing concentrations of sucrose $\left(30-300 \times 10^{-3} \mathrm{M}\right)$ decreased the fraction of multivesicular and multilamellar giant vesicles, while increasing the fraction of unilamellar giant vesicles (Figure 1D-G). The optimum sucrose concentration of $300 \times 10^{-3} \mathrm{M}$ predominantly induced the formation of unilamellar GUVs (Figure 1C, Figure S1A, Supporting Information) and thus was further used for generating multicompartments. According to cryonic transmission electron microscopy (cryo-TEM) images, the membrane thickness of the extruded synthetic GUVs is $\approx 11 \mathrm{~nm}$ (Figure S1D, Supporting Information). To investigate the accessibility of the receptor-like molecule heparin on the GUVs membrane, we examined its interaction with protamine, a positively charged peptide known to bind to the negatively charged heparin. ${ }^{[39]}$ Empty GUVs assembled in the presence and absence of $\mathrm{PDMS}_{65}$-b-hep were first mixed with Bodipy630/650, a fluorescent hydrophobic molecule that aids in the visualization of formed GUVs, and then with Oregon Green 488 (OG488) labeled protamine. CLSM micrographs of individual GUVs reveal colocalization of OG488-protamine and Bodipy630/650 fluorescent signals only at the membrane of GUVs containing $\mathrm{PDMS}_{65}$ - $b$-hep (Figure 1H-I, Figure S2, Supporting Information). This indicates a specific membrane binding interaction between heparin-exposing GUVs and the peptide, while there was no unspecific binding between heparin-free GUVs and the peptide. By mimicking cell membrane receptor-exposure, we now have a tool to probe heparin-binding interactions with various biologically active ligands that will support further development of such GUVs for medical applications.

We then loaded nanoassemblies into GUVs with the intention of creating a multicompartmentalized architecture (Figure 2A). Depending on the chemical nature of the copolymers and their molecular properties, various nanoassemblies were formed: i) reduction-sensitive nanoparticles

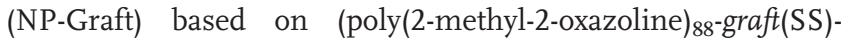
poly $(\varepsilon \text {-caprolactone })_{238} \quad\left(\mathrm{PMOXA}_{88^{-}} \mathrm{g}(\mathrm{SS})-\mathrm{PCL}_{238}\right)$, ii) nonsensitive nanoparticles (NP-Control) based on poly(2-methyl-2oxazoline $)_{30}$-block-poly( $\varepsilon$-caprolactone $)_{62} \quad\left(\mathrm{PMOXA}_{30}-b\right.$ - $\left.\mathrm{PCL}_{62}\right)$, iii) micelles resulting from $\mathrm{PDMS}_{65}-b$-heparin (M100), and iv) polymersomes formed from a mixture of $\mathrm{PMOXA}_{5}-b-\mathrm{PDMS}_{58^{-}}$

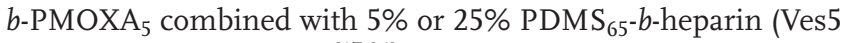
and Ves25, respectively). ${ }^{[17,34]}$ We investigated the architecture and size of these nanoassemblies by a combination of transmission electron microscopy (TEM) and dynamic light scattering (DLS). TEM micrographs indicated the formation of spherical nanoparticles and DLS revealed hydrodynamic diameters of $52 \pm 23 \mathrm{~nm}$ (for NP-Graft), and $104 \pm 40 \mathrm{~nm}$ (for NP-Control). The hydrodynamic diameter of micelles M100 was $99 \pm 34 \mathrm{~nm}$, while polymersomes had diameters of $142 \pm 55 \mathrm{~nm}$ (for Ves5) and $159 \pm 57 \mathrm{~nm}$ (for Ves25), in agreement with our previous reports. ${ }^{[17,34]}$

Hydrophobic Bodipy630/650 was entrapped in the core of both types of nanoparticles (NP-Graft and NP-Control), while OG488 was incorporated in M100, Ves5, and Ves25 to allow their investigation by fluorescence correlation spectroscopy (FCS). 

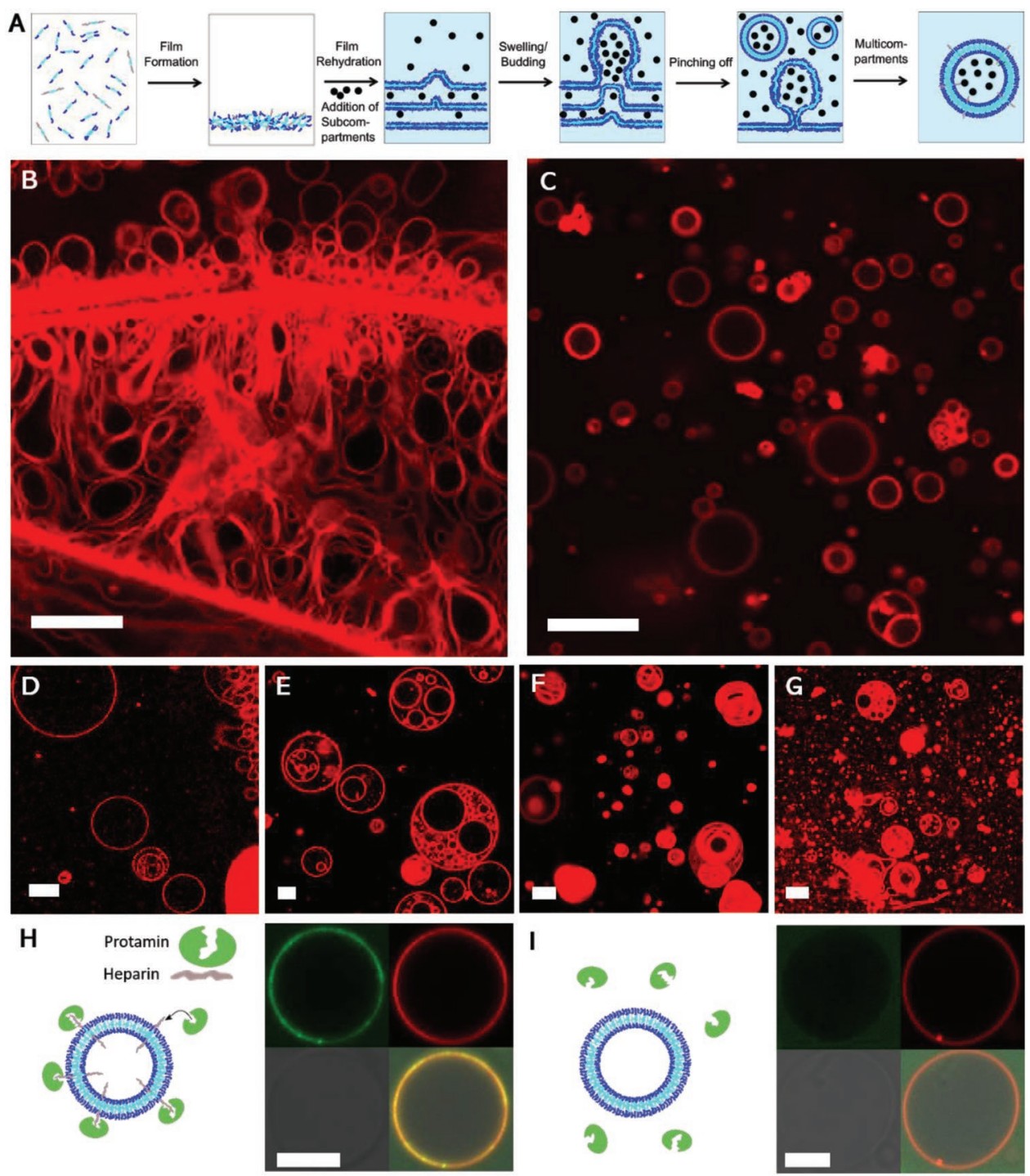

Figure 1. Self-assembly of giant vesicles using film rehydration method. A) Illustration of the formation of multicompartments. The block copolymer mixture was dissolved in an organic solvent, which was evaporated to form a thin dry polymer film. Subsequently, a rehydration buffer including smaller nanocompartments (black dots) and sucrose was added to induce self-assembly. The swollen film started to form buds followed by pinching-off of the buds to form multicompartments. B-G) CLSM images of GUVs. To enhance visualization of the structures, we added the hydrophobic fluorophore Bodipy630/650 (red) that inserts into the vesicle membrane. GUVs were formed B) directly in the observation chamber of an 8-well plate in $300 \times 10^{-3} \mathrm{M}$ sucrose and $\mathrm{C}-\mathrm{G}$ ) in rehydration solutions with decreasing concentration of sucrose in vials: C) $300 \times 10^{-3} \mathrm{M} \mathrm{sucrose,} \mathrm{D)} 200 \times 10^{-3} \mathrm{M}$ sucrose, E) $100 \times 10^{-3} \mathrm{M}$ sucrose, F) $60 \times 10^{-3} \mathrm{M}$ sucrose, and G) $30 \times 10^{-3} \mathrm{M}$ sucrose. Scale bar, $20 \mu \mathrm{m}$. H,I) Interaction of protamine-OC488 (green) with the heparin-decorated and heparin-free giant vesicles (red). Scale bars, $5 \mu \mathrm{m}$.

Synthetic multicompartments were formed by slowly adding a $300 \times 10^{-3} \mathrm{M}$ sucrose solution containing fluorescently labeled nanoassemblies to a polymer film $\left(\mathrm{PMOXA}_{5}-b\right.$ $\mathrm{PDMS}_{58}-b-\mathrm{PMOXA}_{5}$ with $8 \mathrm{wt} \% \mathrm{PDMS}_{65}$-b-heparin) followed by rehydration overnight at $22{ }^{\circ} \mathrm{C}$. Each type of nanoassembly (nanoparticles, micelles, and polymersomes) was separately loaded into GUVs $(4-50 \mu \mathrm{m})$ and investigated by in situ FCS to directly measure their dynamics inside the giant vesicles, prior localized by CLSM (Figure 2B). In FCS, the fluctuation of the fluorescence intensity of fluorophores due to Brownian motion is measured in the femtoliter-sized confocal volume. ${ }^{[40]}$ Freely diffusing fluorescent molecules can be distinguished from fluorophores inserted in membranes or encapsulated in nanoassemblies by a difference in their diffusion times. ${ }^{[11,42]} \mathrm{As}$ the autocorrelation function analysis allows for an estimation of the hydrodynamic diameter of the fluorescent molecules/ assemblies passing through the confocal volume, we used it to follow the nanoassemblies located inside GUVs. We placed the small confocal volume (about $300 \mathrm{~nm} x-y$ dimension and $1 \mu \mathrm{m}$ height ${ }^{[40]}$ inside the cavity of the GUV to precisely measure the presence, size, and stimuli-responsiveness of the nanoassemblies serving as subcompartments (Figure 2B). Only unilamellar, stable, and nonmoving giant vesicles with a minimum diameter of $4 \mu \mathrm{m}$ were selected for FCS measurements. Smaller GUVs were not suitable, because the center of the vesicle was not distinguishable from the membrane and 

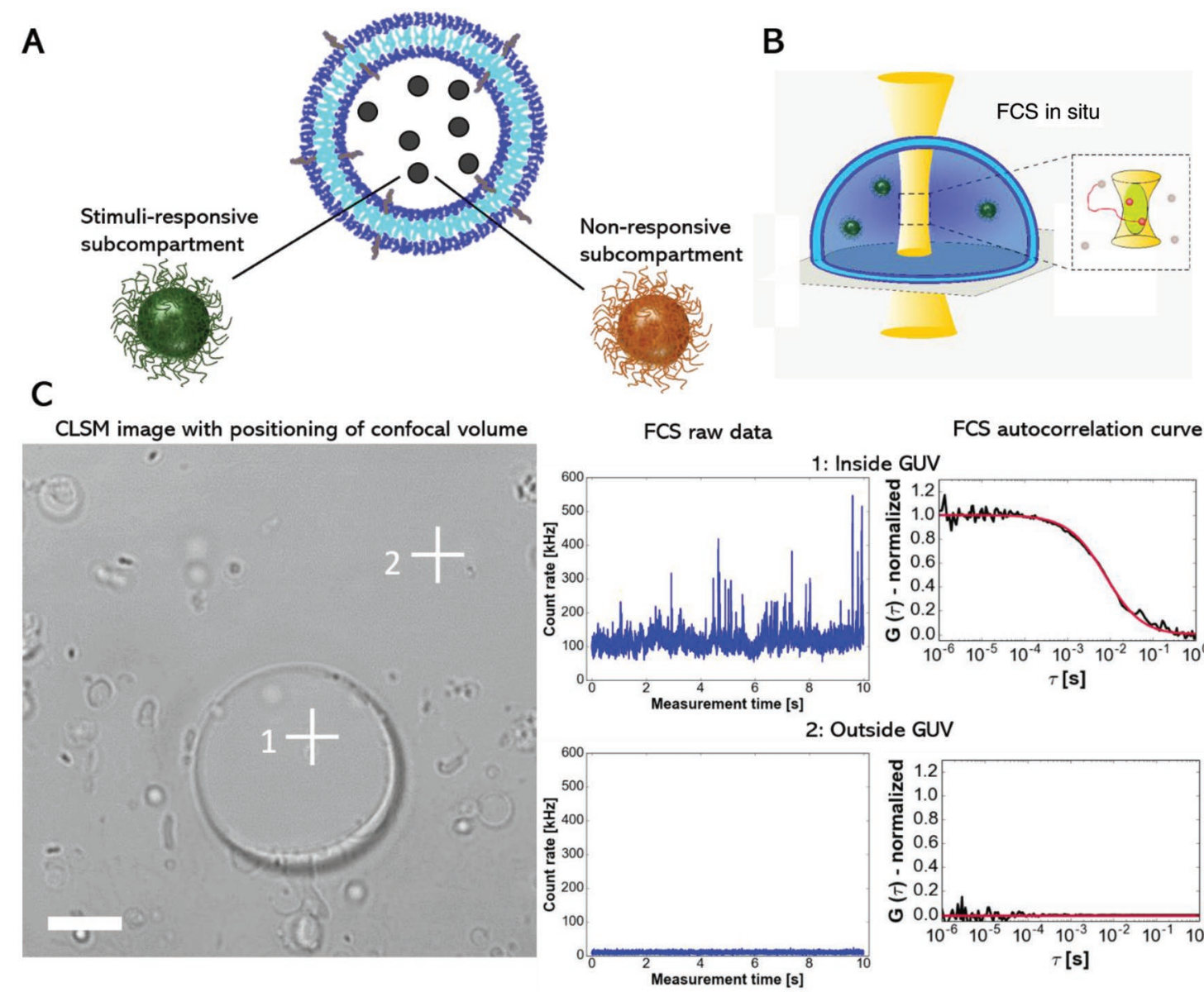

FCS autocorrelation curve

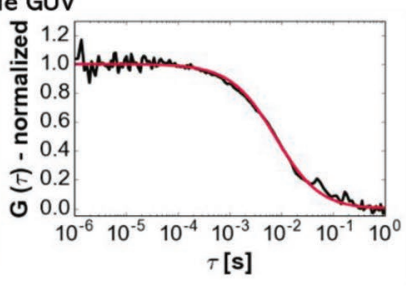

: Outside GUV

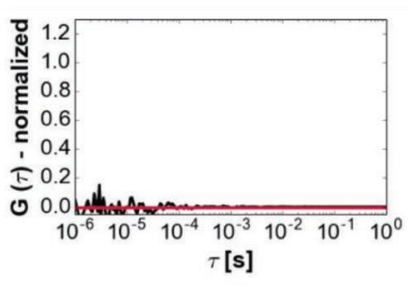

Figure 2. Schematic illustration and characterization of multicompartments. A) Multicompartment, where subcompartments (e.g., stimuli-responsive NP-Graft or nonresponsive NP-Control) are loaded into a polymeric giant vesicle. B) In depth in situ analysis of subcompartments in a giant vesicle with FCS/CLSM by localizing the confocal volume into the GUV cavity. C) FCS measurement in combination with CLSM mode of subcompartments in giant vesicles and its surrounding solution. FCS raw data and autocorrelation curves inside and outside of the NP-Graft loaded giant vesicle. Diffusion time inside the GUV was $5257 \pm 1517 \mu$ s; outside measurement gave no correlation curve indicating diffusing fluorescent species is at a concentration below the detection limit. Count rate inside was $101 \pm 9 \mathrm{kHz}$ and outside was $0.7 \pm 0.5 \mathrm{kHz}$. Scale bar, $10 \mu \mathrm{m}$.

the confocal volume approaches the size of the aqueous inner compartment. As we precisely positioned the confocal volume inside the cavity of the GUVs, the removal of nonencapsulated nanoassemblies was not necessary for FCS measurements and was disregarded by the space precision (Figure 2C). In addition, the concentration of the nonencapsulated nanoassemblies in the surrounding solution of the GUVs, after the dilution required for FCS measurements, was below the detection limit. NP-Graft diffused inside GUVs with a diffusion time of $5257 \pm 1517 \mu \mathrm{s}$ (average count rate of $101 \pm 9 \mathrm{kHz}$ ), while measurements performed outside of the GUVs did not yield an autocorrelation curve. Only background signal was detected (average count rate $=0.7 \pm 0.5 \mathrm{kHz}$ ), confirming the encapsulation of the subcompartments inside the cavity of GUVs and no nonencapsulated subcompartment were detectable in the surrounding GUV solution.

To compare the dynamics of free or encapsulated nanoassemblies, the diffusion times of free dye and free nanoassemblies in solution were compared to those inside of multicompartment GUVs (Figure 3A-E). The autocorrelation curves of encapsulated nanoassemblies were similar to those of freely moving nanoassemblies in solution, as their diffusion times were preserved upon encapsulation. Consistently, the hydrodynamic diameters calculated for the nanoassemblies inside GUVs were comparable to the DLS data obtained from the free nanoassemblies in solution (Figure 3F). This data is in agreement with the values reported previously. ${ }^{[17,34]}$ The subcompartments freely diffused within the lumen of the GUVs and did not aggregate inside. In addition, the subcompartments remained intact within the GUVs and preserved their architecture at least six months (Figure S3A,B, Supporting Information). We also estimated the encapsulation efficiency by analyzing 40 giant vesicles in PBS. According to our FCS data, in $95 \%$ of measured giant vesicles, we found loaded NP-Graft (Figure S3C, Supporting Information).

\subsection{Triggerable Multicompartments with Reduction-Sensitive Inner Subcompartments}

In order to achieve stimuli-responsive multicompartments mimicking a simple signaling reaction as in cells, we 
A
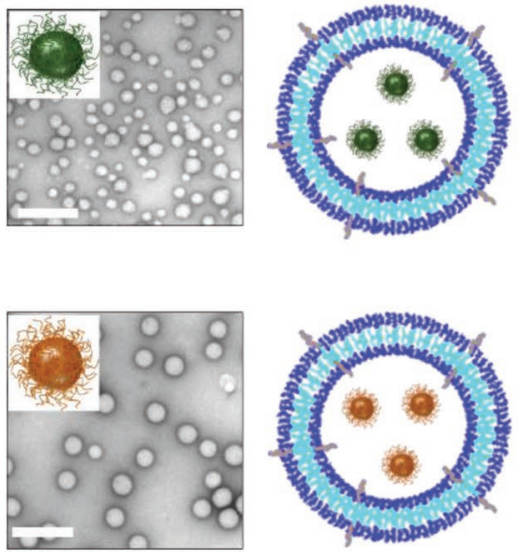

C
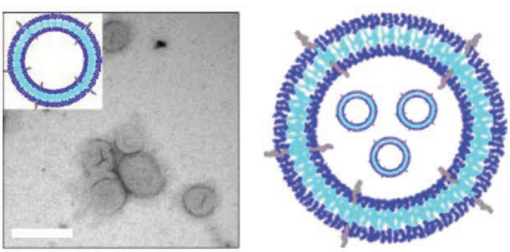

D
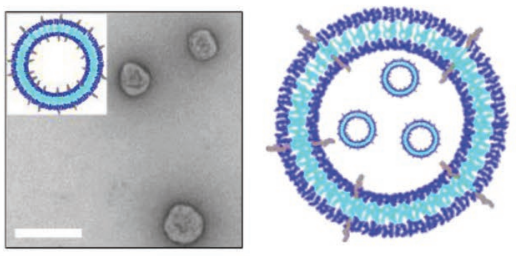

E
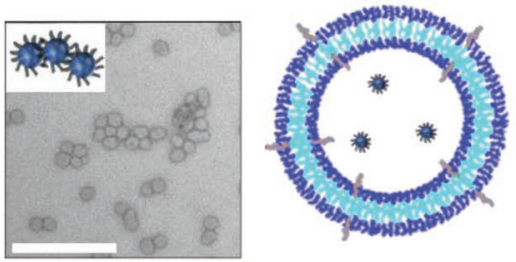
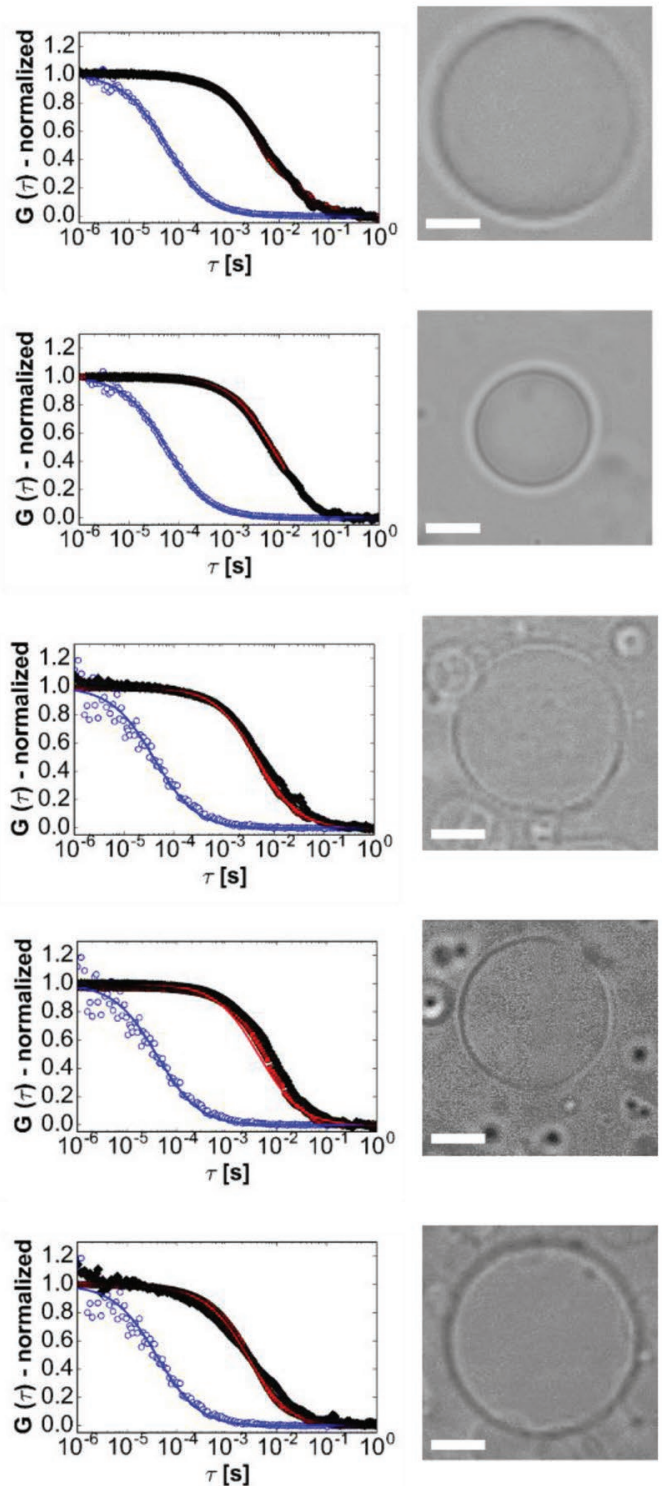

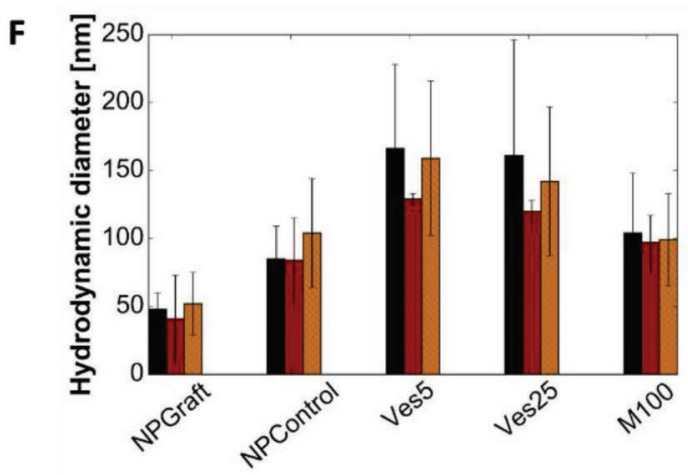

Figure 3. Characterization of multicompartments. A-E) Transmission electron micrograph and schematic illustration of nanoassemblies followed by schematic illustration of multicompartment (left column). FCS measurement in combination with CLSM mode of subcompartments inside giant vesicles (right column). For the FCS measurements: normalized autocorrelation curves are shown with symbols, corresponding fits as solid lines, measurements of the free dye in blue, the nanoassemblies inside giant vesicles in black, and the free nanostructures in solution in red (right column). A) NP-Graft, B) NP-Control, C) Ves5 (5\%-Heparin vesicles), D) Ves25 (25\%-Heparin vesicles), and E) M100 (100\%-Heparin micelles) loaded in giant vesicles. Scale bar, $200 \mathrm{~nm}$ for TEM and $5 \mu \mathrm{m}$ for CLSM images. F) Comparison of the hydrodynamic diameter determined from FCS measurements of nanostructures inside giant vesicles (black bars), in solution (red bars), and from DLS measurements of nanostructures in solution (orange bars). Values are indicated as mean \pm standard deviation. 
A

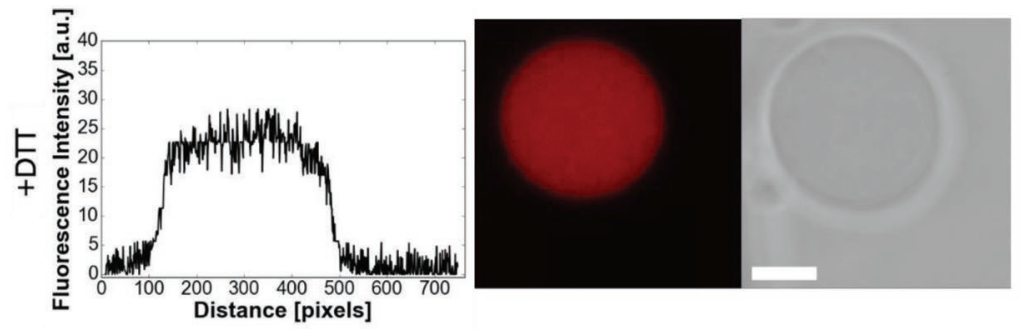

B
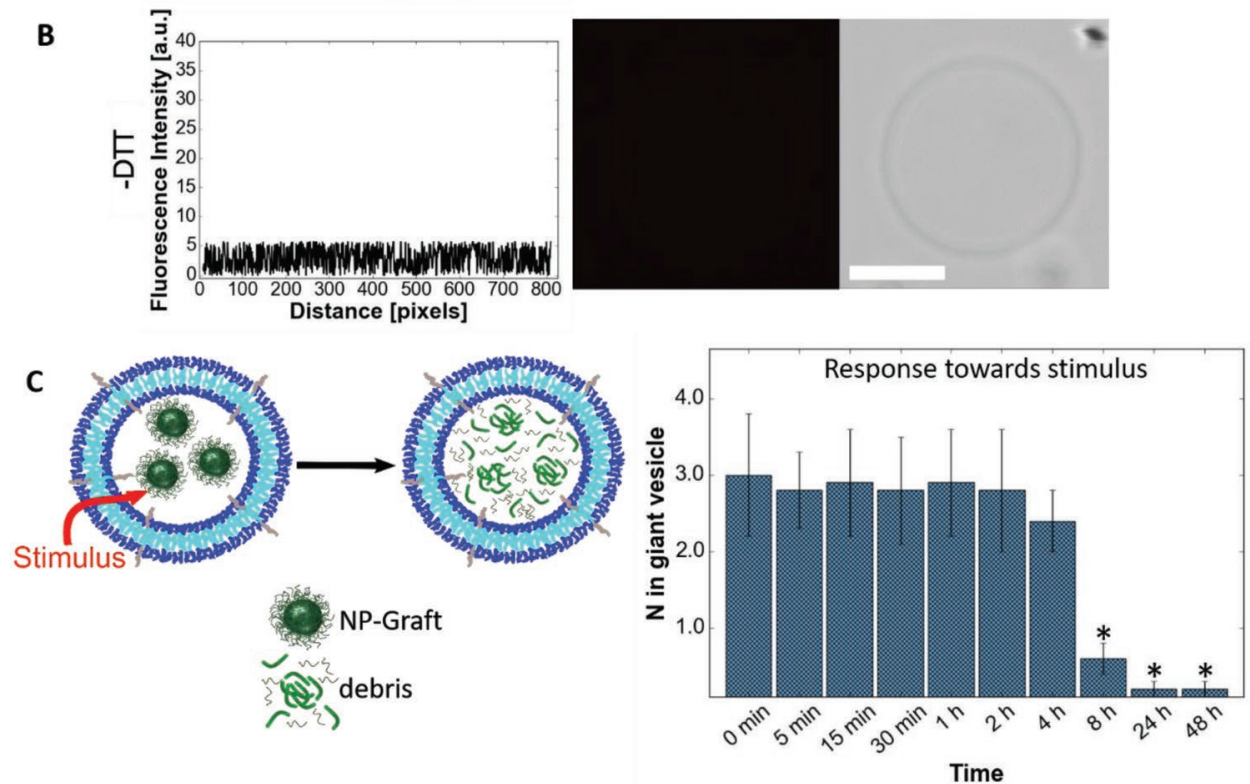

Figure 4. Reduction responsiveness of multicompartments in absence of biopores or ion channel. A,B) Resazurin loaded giant vesicles analyzed by CLSM. Plot profile of the fluorescence intensity along the diagonal of the image (left), CLSM image of resazurin filled giant vesicles (middle) and corresponding bright field image (right): A) In the presence of $10 \times 10^{-3} \mathrm{M}$ DTT after $2 \mathrm{~h}$ incubation and B) in the absence of reducing agents after $2 \mathrm{~h}$ incubation. C) Nanocompartments loaded in giant vesicles. The signaling cascade is illustrated by the schematic. The number of particles $(N)$ in the confocal volume (blue bars) of NP-Graft in giant vesicles were studied by FCS with CLSM mode. Mean of 40 giant vesicles per time point were measured during a $48 \mathrm{~h}$ period in presence of $10 \times 10^{-3} \mathrm{M}$ DTT. Statistical comparison of $N$ at various time points was performed using one-way ANOVA followed by Tukey's HSD test, * $p<0.05$. Significant difference relative to time point at $0 \mathrm{~min}$. Scale bars, $5 \mu \mathrm{m}$.

encapsulated stimuli-responsive subcompartments (NP-Graft) inside GUVs with the aim of inducing their disassembly in the presence of a specific external stimulus.

First, we evaluated the controlled diffusion of reducing agents (external stimulus) through the polymer membrane of GUVs by encapsulating a reduction-sensitive fluorophore resazurin (RZ) as a reporter compound inside GUVs. RZ is converted into the fluorescent resorufin product under reducing conditions (Figure 4A, Figure S4A,B, Supporting Information), while it is nonfluorescent in nonreducing environment (Figure 4B, Figure S4C, Supporting Information). We added DTT or glutathione (GSH) as reducing agents to the outside of RZ-loaded GUVs, at specific concentrations similar to the physiological concentration of GSH in the cell cytosol. ${ }^{[43,44]}$ The fluorescence intensity, associated with resorufin production, increased already $2 \mathrm{~h}$ after addition of DTT, indicating that the reductive agent diffused through the polymer membrane and converted RZ inside GUVs with and without the exposed heparin (Figure S4A,B, Supporting Information). On the contrary, GSH showed no effect even after $72 \mathrm{~h}$ (Figure S4D, Supporting Information), most probably due to its higher molecular weight and charged residues. ${ }^{[45]}$ This behavior is in agreement with reports indicating that exogenous tripeptide GSH does not penetrate the cell membrane, whereas the reducing agent DTT can cross it, ${ }^{[45-48]}$ highlighting a similarity between natural and synthetic polymer based membranes. We achieved the selective diffusion of DTT across the polymer membrane, otherwise impermeable to other molecules with higher molecular weight. We then encapsulated NPGraft nanoparticles as subcompartments inside GUVs and studied the action of external DTT on their integrity when loaded with Bodipy630/650 as model molecule. Molecular brightness (counts per molecule) values obtained by FCS measurements were used to get an estimate for number of Bodipy630/650 per NP-Graft, which resulted in 46 dye molecules per NP-Graft.

Time-resolved FCS measurements performed inside GUVS revealed that addition of DTT to multicompartments induced a significant decrease in the number of Bodipy630/650-loaded NP-Graft particles inside the observation volume to almost 0 after $24 \mathrm{~h}$ incubation (average of $40 \mathrm{GUVs} /$ time point, 
Figure 4C). The disulfide bonds that connect hydrophilic and hydrophobic polymers of NP-Graft were cleaved and induced the rupture of NP-Graft subcompartments. In addition, a significant decrease in the count rate was observed after $24 \mathrm{~h}$ incubation with $10 \times 10^{-3}$ м DTT (Figure S5A,B, Supporting Information). This decrease can be attributed to the migration of the hydrophobic Bodipy630/650 toward the GUV's membrane, once released upon disintegration of the NP-Graft. As expected, the nanoparticle disassembly was delayed when encapsulated within GUVs due to the additional barrier (GUV membrane). Indeed, in solution, the disassembly of NP-Graft nanoparticles and their content release was complete within $90 \mathrm{~min},{ }^{[17]}$ whereas it took $24 \mathrm{~h}$ to reach $90 \%$ for NP-Graft as subcompartments within GUVs. More specifically, in an average GUV volume of $1112 \pm 407 \mu^{3}$, the average nanoparticle number of $4082 \pm 1051$ initially encapsulated dropped to $80 \pm 23$ after $48 \mathrm{~h}$ in presence of DTT (Figure S7A, Supporting Information). The triggered structural change of these subcompartments was successfully observed in individual GUVs by FCS. The presence of negatively charged heparin molecules on the polymer membrane did not impact the cleavage of the disulfide bonds of the NP-Graft inside the multicompartment system (Figures S5B,S6A, Supporting Information). When no reducing agent was added, NP-Graft subcompartments preserved their integrity inside GUVs (Figures S6C,S5F, Supporting Information). According to the histogram, the size distribution of the NP-Graft loaded GUVS used for FCS measurements before addition of DTT was between 7 and $33 \mu \mathrm{m}(N=40$ GUVs, Figure S7B, Supporting Information). In general, the simple and rapid formation of multicompartments via gentle film rehydration method provided a polydisperse mixture of sizes in the range between 2 to $40 \mu \mathrm{m}(N=400$ NP-Graft-loaded GUVs, Figure S7C, Supporting Information). Only GUVs with sizes above $4 \mu \mathrm{m}$, where the aqueous core can clearly be distinguished from the membrane, were chosen for FCS and CLSM analysis of the functionality of the assemblies. If a better control of the size distribution is needed, one can use a patterned surface for film rehydration ${ }^{[49]}$ or transfer the system to a microfluidic-based formation method. ${ }^{[31,32]}$ As expected, GSH had no observable effect on encapsulated NP-Graft (Figures S5D,S6B, Supporting Information). No GUV swelling was observed after DTT addition, excluding a dilution effect (Figure S7A, Supporting Information).

As NP-Control nanoparticles were not stimuli-responsive, they were not affected by the presence of the reducing agent, and the multicompartments remained intact (Figures S5C,E,G and S6D-F, Supporting Information). Therefore, we could conclude that the disassembly of the encapsulated NP-Graft nanoparticles proceeded via cleavage of disulfide bonds between PMOXA domains and PCL blocks upon addition of DTT as signaling molecule from the surrounding environment of the GUVs. When the subcompartments are nonresponsive or the external stimulus is not present in the lumen, the multicompartments preserve their architecture and remain in a "silent mode" for several months (Figure S3, Supporting Information). This behavior can be compared to a molecular AND gate ${ }^{[21]}$ as the dye release only happens in presence of a specific reducing agent (DTT) and NP-Graft.

\subsection{Triggered Enzymatic Reactions in Multicompartments}

In order to increase the complexity of the stimuli-responsive multicompartments and trigger a desired reaction, we coencapsulated enzymes and NP-Graft nanoparticles loaded with their corresponding substrates within GUVs. We then controlled the triggered subcompartment's destruction by applying an external signal to switch on the enzymatic reaction. The straightforward production and the mild conditions for the formation of GUVs make them compatible with biomolecules thus allowing us to encapsulate enzymes. We used as an enzyme model, pancreatic lipase, which is hydrophobic, and therefore expected to be entrapped in the GUV's membrane. To achieve a controlled and responsive multicompartment, the fluorogenic lipase substrate 1,2-Di-O-lauryl-rac-glycero-3-(glutaric acid 6-methylresorufin ester) (DGGR) ${ }^{[50]}$ was first incorporated in the NP-Graft to segregate it from the enzyme when they were coloaded inside GUVs (Figure 5A). When the reducing agent is added to the exterior medium a three-step pathway is hypothesized to provide the functionality: 1) DTT penetrates inside GUVs, 2) it induces the disintegration of the NP-Graft and the release of DGGR, and 3) the substrate is free to participate in the enzymatic reaction.

In solution, when lipase, DGGR-loaded NP-Graft and DTT were mixed, a significant increase of fluorescence associated with product formation was observed (Figure S8, Supporting Information). This indicates that the substrate entrapped inside NP-Graft was released in the presence of DTT to finally form the fluorescent product by the subsequent lipase activity. When only DGGR-loaded NP-Graft and lipase were mixed in solution, no fluorescence signal was detected indicating that the enzyme and its substrate are completely separated. As a control, DGGRloaded NP-Graft and DTT mixture in absence of lipase, led to a minimal increase of fluorescence in PBS and $300 \times 10^{-3} \mathrm{M}$ sucrose. In presence of the lipase inhibitor Orlistat, lipase was completely inhibited and yielded no product.

Next, we encapsulated DGGR-loaded NP-Graft nanoparticles and lipase within GUVs to obtain multicompartments. The absence of fluorescence after assembly of the multicompartments indicated successful compartmentalization of the substrate with no contact with the enzyme (Figure 5B, Figure S9B, Supporting Information). Upon addition of DTT to the GUVs' environment, the increase in the fluorescent signal associated with the product (methylresorufin) confirms the enzyme activity triggered by the release of the substrate from the sacrificial nanoparticles, as observed by CLSM (Figure 5B, Figure S9A, Supporting Information). In $83 \%$ of GUVs, methylresorufin tended to partition into the membrane (red ring) (Figure S10, Supporting Information). As lipases are known to typically work at the interface between hydrophobic and hydrophilic environments, methylresorufin was produced close to the outer membrane. In $12 \%$ of the vesicles, the membrane and the lumen showed fluorescence (red vesicle), indicating that a fraction of the substrate was activated by lipase and tended to stick to the NP-Graft debris. The remaining $5 \%$ of multicompartments showed no fluorescence. Note that any free, nonencapsulated lipase in the surroundings of the GUVs was inhibited by adding Orlistat to the solution to avoid bias of reactions outside the vesicles. Therefore, no further purification was necessary. Outer membrane of GUVs with incorporated DGGR-loaded NP-Graft and lipase, remained 

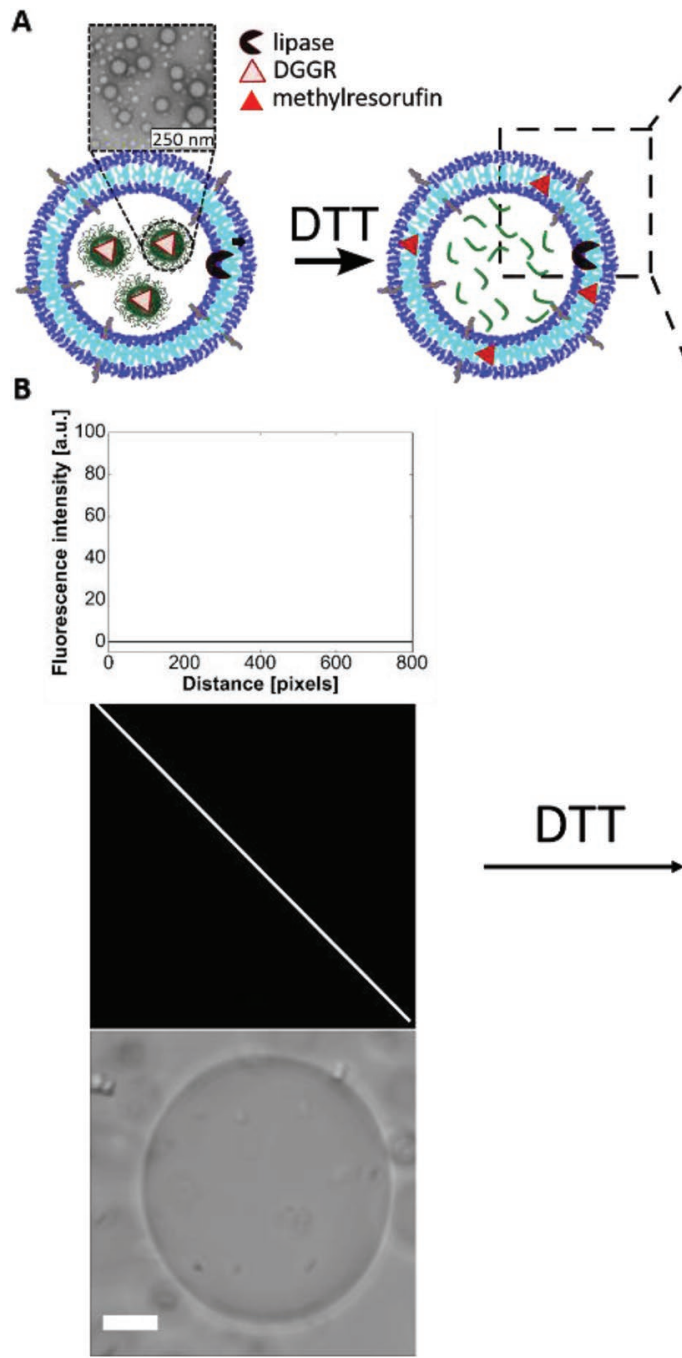

Figure 5. Compartmentalization of enzyme reaction. A) Schematic representation of signaling pathway resulting in lipase activity. The DGGR-loaded NP-Graft and lipase were co-encapsulated in the giant vesicles. The substrate was released in presence of DTT and was transformed by lipase to form the fluorescent product (methylresorufin) which preferentially associated with the giant vesicle membrane. B) CLSM imaging of DGGR-loaded NP-Graft and lipase loaded giant vesicles in absence (left) and presence of DTT (right). Histogram along diagonal of fluorescence image (top), fluorescence image (middle), and bright field image (bottom). Scale bars, $5 \mu \mathrm{m}$.

stable in presence of DTT after $24 \mathrm{~h}$ incubation with a size distribution between 6 and $22 \mu \mathrm{m}$ for measured GUVs $(N=40$ GUVs, Figure S10E, Supporting Information). In conclusion, an enzymatic reaction inside multicompartments was successfully triggered via a cascade involving an external signal that induced a change in the internal architecture (sacrificial subcompartments) and release of the substrate to encounter the encapsulated enzymes. Here we used an enzyme that exhibits its highest catalytic activity at the interface between the hydrophobic membrane of the GUV and hydrophilic content; in general, this type of multicompartment could be used as a tool to study reaction kinetics of hydrophobic enzymes that otherwise would be difficult to study in solution. The molecular logics now rest on a hybrid polymer/enzyme system, with one input still completely synthetic, and the other one biological.

\subsection{Ion Channel Recruitment in Triggerable Multicompartments}

Moving a step further in the development of our stimuli-responsive multicompartment system, we aimed to induce selective membrane permeabilization, for signaling purposes, by recruiting ion channels from internal subcompartments to the GUV membrane. Dynamic ion channel recruitment to the cell membrane to modulate permeability was for example demonstrated in neurons. Endosomal compartments are involved in these processes of reducing or increasing the number of these specific membrane proteins by endocytosis and exocytosis, respectively. ${ }^{[5]}$ As a suitable model, we chose to study the induced passage of monovalent cations through the GUV membrane when mediated by a recruited biopore, the peptide ion channel gramicidin A (gA). ${ }^{[37]}$ The gA ion channel is pre-entrapped in NP-Graft. The signaling pathway from the surrounding environment of multicompartments is based on a primary signal (DTT), inducing a secondary signal (sodium ion flow through the GUV membrane). To achieve this sequence of controlled signals, we first encapsulated gA-loaded NP-Graft as subcompartments within GUVs in combination with a sodium sensitive dye, Asante Natrium Green2 (ANG2) (Figure 6A, Figure S11, Supporting Information). Upon addition of DTT as the first signal, NP-Graft nanoparticles disintegrated and released gA that spontaneously inserted into the GUV membrane due to its hydrophobicity. gA insertion into GUVs' membrane allowed an influx of $\mathrm{Na}^{+}$ions from the environment into the GUVs cavity followed by activation of the $\mathrm{Na}^{+}$sensitive dye. Overall, $85 \%$ of multicompartments were functional and induced the recruitment of biopores into the polymer membranes, as indicated by dye activation (Figure S12, Supporting Information). In around 15\% of multicompartments, no fluorescence was detected, most probably due to an insufficient loading of gA inside NP-Graft, ANG2, or NP-Graft inside GUVs or a combination thereof. According to CLSM images, gA-loaded NP-Graft and ANG2 in GUVs used for FCS measurements in presence of DTT after $24 \mathrm{~h}$ incubation preserved the outer membrane (GUV) with a size distribution between 7 and $22 \mu \mathrm{m}(N=40$ GUVs, Figure S12D, Supporting Information). In the absence of the reducing agent DTT, the ion channels remained entrapped inside subcompartments and the multicompartments remained impermeable to $\mathrm{Na}^{+}$ions: no increase in fluorescence was detected since there was no activation of ANG2. The dye ANG2 is activated only in presence of sodium ions (PBS) (Figure S13, Supporting Information), while in sucrose solution or in HEPES buffer with $10 \times 10^{-3} \mathrm{M}$ 

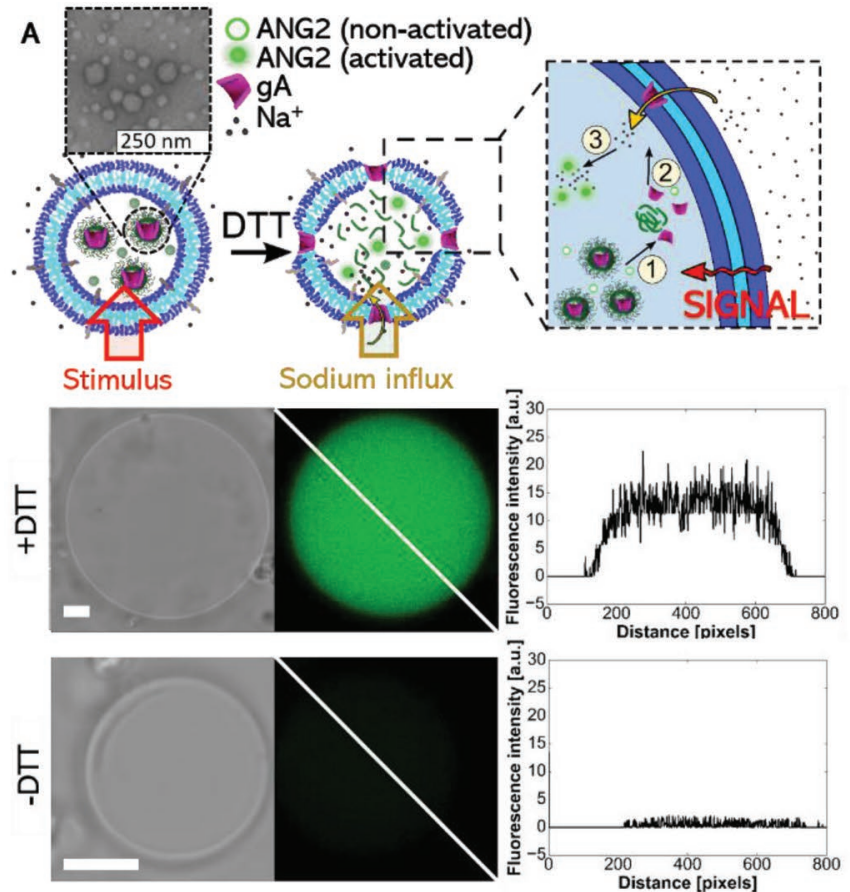

B

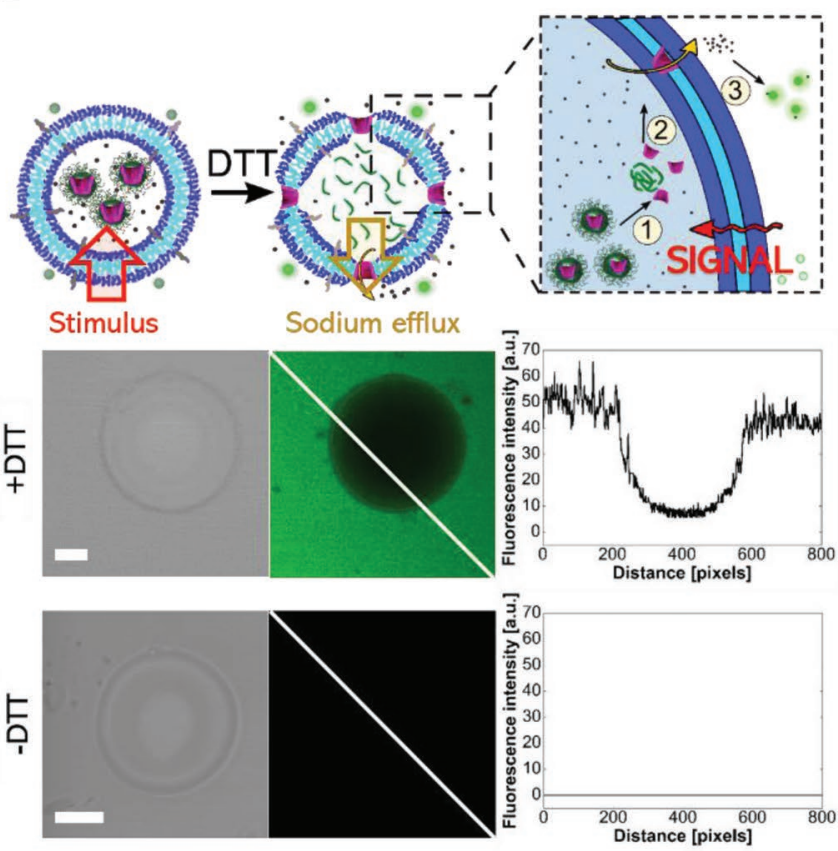

Figure 6. Triggered gramicidin (gA) ion channel recruitment from internal subcompartments to the polymer membrane of a multicompartment using CLSM imaging. A) Schematic representation (top) of gA mediated import of sodium ions. Upon the addition of DTT, encapsulated gA is released from its NP-Graft and inserts into the GUV membrane boundary. This allows sodium ions from the outside to enter the GUV cavity where they activate the sodium sensitive dye ANG2. Bright field image (left), fluorescence image (middle), and histogram along the diagonal of fluorescence image (right) in presence (middle) and absence (bottom) of DTT. B) Schematic representation (top) of gA mediated efflux of sodium ions from the GUV cavity. DTT induced gA permeabilization of the GUV membrane, allows sodium ions in the interior to exit the vesicle and activate the ANG2 in the surrounding environment of the multicompartment. Bright field image (left), fluorescence image (middle) and histogram along the diagonal of fluorescence image (right) in presence (middle) and absence (bottom) of DTT. Scale bars, $5 \mu \mathrm{m}$.

DTT, it remains nonfluorescent. As a control, when NP-Graft nanoparticles without cargo (EmptyNP) were used, the ANG2 dye was not activated inside the GUVs in presence and absence of reducing agent (Figure S14, Supporting Information) due to their impermeability toward sodium ions without ion channel insertion. Therefore, the use of sacrificial subcompartments loaded with desired molecules inside multicompartments represent an elegant manner to provide conditions for sequential signaling pathways.

We then changed the arrangement by inverting the location of the signals in relation to the architecture of the multicompartments: the primary signal (DTT) comes from the environment, while the secondary signals (sodium ions) are subsequently released from the inner cavity of the GUVs to induce a change in the external medium (Figure 6B, Figure S15, Supporting Information). In this respect, we entrapped gA-loaded NP-Graft together with sodium ions in the GUV lumen, while ANG2 dye was added to the multicompartments' environment, in a sodium-free buffer. When DTT was added from the outside, it induced the gA recruitment from the subcompartments and incorporation into the GUV membrane. This resulted in the diffusion of encapsulated sodium ions from the interior of the multicompartments to their exterior where the sodium sensitive dye ANG2 was activated. As expected, the sequence did not occur in the absence of DTT. The triggered recruitment and functional insertion of gA into the membrane of the multicompartments via responsive sacrificial subcompartments showed that we were able to successfully introduce a sequential signaling pathway into our multicompartments. The induced reconstitution of gA allowed the passage of sodium ions "on demand," either from outside or vice versa. Similarly to the enzyme system, the AND gate now combines a hybrid polymer/peptide system. In addition, depending on the setup, a second AND gate could induce the flow of ions.

\section{Conclusion}

Inspired by signaling pathways in cells, we developed here a strategy to auto-trigger changes within micrometric multicompartments and induce specific responses based on signaling cascades. First, multicompartments were generated by encapsulating various subcompartments (micelles, polymersomes, and nanoparticles) inside synthetic GUVs, which remained stable for extended periods of time without in situ aggregation, and studied their assembly in detail by FCS/CLSM. The selective permeability of the compartment membrane toward the reducing agent DTT was then used to induce a reductive milieu in the lumen of GUVs that disassembled the sensitive subcompartments, while no effect was observed for the nonresponsive ones. Desired molecules (enzymes, reporter compounds) and 
corresponding partners (substrates, biopores) entrapped in responsive nanoparticles were simultaneously co-encapsulated within GUVs to obtain a spatial segregation. Upon penetration through the membrane of GUVs, DTT induced the disintegration of the sensitive nanoparticles and subsequent release of their content. The substrates were able to encounter the enzymes and trigger their reaction, while ion channels (gA) were recruited to the GUV's membrane and enabled a constant in/out flow of sodium ions. Our strategy has as advantages a very simple preparation method, high versatility, and straightforward manner to induce auto-controlled sequences of reactions/changes inside microcompartments. As anticipated, our system went beyond pure nanotechnological terms and encroached into molecular logics territory, where the changes in internal architecture led to changes in the GUVs behavior, leading to a simple, self-contained AND gate. The use of semisynthetic materials will have to be further investigated, as it has the potential of bridging the well-known biochemical logics and the growing field of pure molecular logics.

\section{Supporting Information}

Supporting Information is available from the Wiley Online Library or from the author.

\section{Acknowledgements}

The authors gratefully acknowledge financial support from the Swiss National Science Foundation, NCCR-MSE, and University of Basel. The authors thank to S. Kasper for PMOXA-b-PDMS- $b$-PMOXA synthesis, G. Persy for TEM imaging, and Carola Alampi (C-CINA) for cryo-TEM imaging. Dr. C.A. Schönenberger, Dr. E. Konishcheva, and Dr. M. Garni (all University of Basel) are acknowledged for detailed discussions and editing the manuscript.

\section{Conflict of Interest}

The authors declare no conflict of interest.

\section{Keywords}

biomimetic, functional giant vesicles, mimics of signaling pathways, stimuli-responsive multicompartment systems, supramolecular polymeric materials

Received: May 28, 2019

Revised: July 3, 2019

Published online: August 9, 2019

[1] A. Armada-Moreira, B. Thingholm, K. Andreassen, A. M. Sebastião, S. H. Vaz, B. Städler, Adv. Biosyst. 2018, 2, 1700244.

[2] P. Walde, BioEssays 2010, 32, 296.

[3] C. D. Keating, Acc. Chem. Res. 2012, 45, 2114.

[4] M. J. Lee, M. B. Yaffe, Cold Spring Harbor Perspect. Biol. 2016, 8, a005918.

[5] C. J. Groten, N. S. Magoski, J. Neurosci. 2015, 35, 2747.

[6] J. Capera, C. Serrano-Novillo, M. Navarro-Pérez, S. Cassinelli, A. Felipe, Int. J. Mol. Sci. 2019, 20, 734.
[7] E. Balse, S. El-Haou, G. Dillanian, A. Dauphin, J. Eldstrom, D. Fedida, A. Coulombe, S. N. Hatem, Proc. Natl. Acad. Sci. USA 2009, 106, 14681.

[8] E. Balse, H. E. Boycott, Front. Physiol. 2017, 8, 808.

[9] E. Rideau, R. Dimova, P. Schwille, F. R. Wurm, K. Landfester, Chem. Soc. Rev. 2018, 47, 8572.

[10] J.-L. Rigaud, D. Lévy, Methods Enzymol. 2003, 372, 65.

[11] H. C. Shum, Y. Zhao, S.-H. Kim, D. A. Weitz, Angew. Chem. 2011, 123,1686; Angew. Chem., Int. Ed. 2011, 50, 1648.

[12] A. Najer, D. Wu, D. Vasquez, C. G. Palivan, W. Meier, Nanomedicine 2013, 8, 425.

[13] A. Belluati, I. Craciun, C. E. Meyer, S. Rigo, C. G. Palivan, Curr. Opin. Biotechnol. 2019, 60, 53.

[14] T. Einfalt, D. Witzigmann, C. Edlinger, S. Sieber, R. Goers, A. Najer, M. Spulber, O. Onaca-Fischer, J. Huwyler, C. G. Palivan, Nat. Commun. 2018, 9, 1127.

[15] H. Gumz, S. Boye, B. lyisan, V. Krönert, P. Formanek, B. Voit, A. Lederer, D. Appelhans, Adv. Sci. 2019, 6, 1801299.

[16] K. T. Kim, J. J. L. M. Cornelissen, R. J. M. Nolte, J. C. M. van Hest, Adv. Mater. 2009, 21, 2787.

[17] A. Najer, D. Wu, M. G. Nussbaumer, G. Schwertz, A. Schwab, M. C. Witschel, A. Schäfer, F. Diederich, M. Rottmann, C. G. Palivan, H.-P. Beck, W. Meier, Nanoscale 2016, 8, 14858.

[18] T. Einfalt, R. Goers, I. A. Dinu, A. Najer, M. Spulber, O. OnacaFischer, C. G. Palivan, Nano Lett. 2015, 15, 7596.

[19] C. Martino, A. J. deMello, Interface Focus 2016, 6, 20160011.

[20] A. X. Lu, H. Oh, J. L. Terrell, W. E. Bentley, S. R. Raghavan, Chem. Sci. 2017, 8, 6893.

[21] A. P. de Silva, S. Uchiyama, Nat. Nanotechnol. 2007, 2, 399.

[22] E. U. Akkaya, E. Katz, U. Pischel, ChemPhysChem 2017, 18, 1665.

[23] A. Peyret, E. Ibarboure, N. Pippa, S. Lecommandoux, Langmuir 2017, 33, 7079.

[24] L. Wei, S. Yan, H. Wang, H. Yang, NPG Asia Mater. 2018, 10, 899.

[25] S. Utech, C. Scherer, M. Maskos, J. Magn. Magn. Mater. 2009, 321, 1386.

[26] H. Lomas, A. P. R. Johnston, G. K. Such, Z. Zhu, K. Liang, M. P. V. Koeverden, S. Alongkornchotikul, F. Caruso, Small 2011, 7, 2109.

[27] C. D. Driever, X. Mulet, L. J. Waddington, A. Postma, H. Thissen, F. Caruso, C. J. Drummond, Langmuir 2013, 29, 12891.

[28] X. Liu, P. Formanek, B. Voit, D. Appelhans, Angew. Chem. 2017, 129, 16451; Angew. Chem., Int. Ed. 2017, 56, 16233.

[29] L. Hosta-Rigau, S. F. Chung, A. Postma, R. Chandrawati, B. Städler, F. Caruso, Adv. Mater. 2011, 23, 4082.

[30] J. W. Hindley, Y. Elani, C. M. McGilvery, S. Ali, C. L. Bevan, R. V. Law, O. Ces, Nat. Commun. 2018, 9, 1093.

[31] H. Wang, Z. Zhao, Y. Liu, C. Shao, F. Bian, Y. Zhao, Sci. Adv. 2018, 4, eaat2816.

[32] L. Shang, Y. Cheng, Y. Zhao, Chem. Rev. 2017, 117, 7964.

[33] K. Kamiya, S. Takeuchi, J. Mater. Chem. B 2017, 5, 5911.

[34] A. Najer, D. Wu, A. Bieri, F. Brand, C. G. Palivan, H.-P. Beck, W. Meier, ACS Nano 2014, 8, 12560.

[35] A. Najer, S. Thamboo, J. T. Duskey, C. G. Palivan, H.-P. Beck, W. Meier, Macromol. Rapid Commun. 2015, 36, 1923.

[36] A. Najer, S. Thamboo, C. G. Palivan, H.-P. Beck, W. Meier, CHIMIA Int. J. Chem. 2016, 70, 288

[37] M. Lomora, M. Garni, F. Itel, P. Tanner, M. Spulber, C. G. Palivan, Biomaterials 2015, 53, 406.

[38] B. L. Farrugia, Y. Mi, H. N. Kim, J. M. Whitelock, S. M. Baker, W. P. Wiesmann, Z. Li, P. Maitz, M. S. Lord, Adv. Funct. Mater. 2018, 28, 1802818.

[39] J. C. van Kerkhof, P. Bergveld, R. B. M. Schasfoort, Biosens. Bioelectron. 1993, 8, 463.

[40] N. Altan-Bonnet, G. Altan-Bonnet, Curr. Protoc. Cell Biol. 2009, 45, 4.24.1.

[41] F. Itel, A. Najer, C. G. Palivan, W. Meier, Nano Lett. 2015, 15, 3871. 
[42] F. Itel, M. Chami, A. Najer, S. Lörcher, D. Wu, I. A. Dinu, W. Meier, Macromolecules 2014, 47, 7588.

[43] A. Meister, Trends Biochem. Sci. 1988, 13, 185

[44] B. Ates, B. C. Ercal, K. Manda, L. Abraham, N. Ercal, Biomed. Chromatogr. 2009, 23, 119.

[45] W.-S. Chung, J. M. Farley, H. A. Drummond, Cell. Physiol. Biochem. 2011, 27, 129 .
[46] P. Graf, H. Sies, Biochem. Pharmacol. 1984, 33, 639.

[47] J. A. Holme, D. C. Dahlin, S. D. Nelson, E. Dybing, Biochem. Pharmacol. 1984, 33, 401.

[48] D. M. Ziegler, Annu. Rev. Biochem. 1985, 54, 305.

[49] J. R. Howse, R. A. L. Jones, G. Battaglia, R. E. Ducker, G. J. Leggett, A. J. Ryan, Nat. Mater. 2009, 8, 507.

[50] M. Panteghini, R. Bonora, F. Pagani, Ann. Clin. Biochem. 2001, 38, 365. 